Wichtige sicherheitsrelevante Information | Baar, 25. Februar 2020

Firmagon ${ }^{\circledR}$ 120/80 mg Pulver und Lösungsmittel zur Herstellung einer Injektionssuspension, ZL-Nummer 59455 Betroffene Chargen: P17685C, P17686C, R11766C, R14363H, R11860G

\title{
Vorsorglicher Chargenrückruf bis auf Stufe Detailhandel
}

Sehr geehrte Frau Doktor, sehr geehrter Herr Doktor

Hiermit informieren wir Sie in Absprache mit Swissmedic über einen vorsorglichen Chargenrückruf von Firmagon ${ }^{\circledR}$ 120/80 mg Pulver und Lösungsmittel zur Herstellung einer Injektionssuspension gemäss der Tabelle hier unten, bis auf Stufe Detailhandel.

Vom Rückruf betroffen sind ausschliesslich die in der folgenden Tabelle erwähnten Chargen und Packungsgrössen:

\begin{tabular}{|l|l|l|l|l|}
\hline & Pharmacode & Lot number & $\begin{array}{l}\text { Manufactur- } \\
\text { ing date }\end{array}$ & Expiry date \\
\hline \multirow{4}{*}{ Firmagon ${ }^{\oplus} 80 \mathrm{mg}$} & \multirow{3}{*}{5666436} & P17685C & 11-Jan-2019 & 30-Apr-2021 \\
\cline { 3 - 5 } & & P17686C & 11-Jan-2019 & 30-Apr-2021 \\
\cline { 3 - 5 } & & R11766C & 02-Apr-2019 & 31-Jan-2022 \\
\cline { 3 - 5 } & & R14363H & 16-Aug-2019 & 31-Jul-2022 \\
\hline Firmagon ${ }^{\oplus} 120 \mathrm{mg}$ & 5666442 & R11860G & 12-Apr-2019 & 31-Jan-2022 \\
\hline
\end{tabular}

\section{Grund für den Rückruf:}

Der Rückruf erfolgt aufgrund des Nachweises von Silikonöl aus dem Kühlsystem eines Herstellungsbehälters im Wirkstoff Degarelix.

Für spezifische Patientengruppen (z.B. Patienten mit Metastasen/ Patienten mit schwerer koronarer kardiovaskulärer Erkrankung) gilt es, das medizinische Risiko eines Wechsels der Therapie durch den behandelnden Arzt in Absprache mit dem Patienten dem Risiko einer Applikation einer kontaminierten Einheit Firmagon ${ }^{\circledR}$ gegenüberzustellen.

Bitte überprüfen Sie Ihre Bestände und senden Sie die betroffenen Chargen auf dem umgekehrten Lieferweg zurück. Falls Sie die Ware beim Grossisten bestellt haben, bitten wir Sie, diese an den Grossisten zurückzusenden.
Sie werden für die retournierte Ware eine Gutschrift erhalten, bis neue, konforme Ware ca. Ende März 2020 zur Verfügung steht.

Für Meldungen über unerwünschte Arzneimittelwirkungen (UAW) empfiehlt Swissmedic, das dafür entwickelte Meldeportal zu verwenden. Mit dem sogenannten Electronic Vigilance System (EIViS) können UAW direkt oder durch Hochladen einer XML-Datei erfasst werden. Alle erforderlichen Informationen sind zu finden unter www.swissmedic.ch $\rightarrow$ Humanarzneimittel $\rightarrow$ Marktüberwachung $\rightarrow$ Pharmacovigilance

Wir entschuldigen uns für die entstandenen Unannehmlichkeiten in Zusammenhang mit dem Rückruf von Firmagon ${ }^{\circledR}$ 120/80 mg und danken Ihnen im Voraus für Ihr Verständnis und Ihre Mitwirkung.

Für weitere Fragen stehen wir Ihnen unter der Telefonnummer +4141 7689696 oder der E-Mail-Adresse info@ferring.ch gerne zur Verfügung.

Freundliche Grüsse

Ferring $A G$

Dr. Sraboni Ghose

Kristien Vanthuyne

Fachtechnisch verantwortliche Person
Geschäftsführerin 